\title{
Phronesis
}

\section{La place de la recherche en sciences humaines, sociales et économiques dans les écoles d'ingénieurs}

\section{Bilan et perspectives}

\section{Michel Sonntag, François Gitzhofer et Michel Lejeune}

Volume 4, numéro 2, 2015

La place de la recherche en sciences humaines, sociales et

économiques dans les écoles d'ingénieurs : bilan et perspectives

URI : https://id.erudit.org/iderudit/1033445ar

DOI : https://doi.org/10.7202/1033445ar

Aller au sommaire du numéro

Éditeur(s)

Université de Sherbrooke

ISSN

1925-4873 (numérique)

Découvrir la revue

Citer ce document

Sonntag, M., Gitzhofer, F. \& Lejeune, M. (2015). La place de la recherche en sciences humaines, sociales et économiques dans les écoles d'ingénieurs : bilan et perspectives. Phronesis, 4(2), 1-4. https://doi.org/10.7202/1033445ar 


\section{La place de la recherche en sciences humaines, sociales et économiques dans les écoles d'ingénieurs}

Numéro coordonné par:

Michel SONNTAG, INSA, Strasbourg, France

François GITZHOFER, Faculté de génie, Université de Sherbrooke, Canada

Michel LEJEUNE, École polytechnique de Montréal, Canada

\section{BILAN ET PERSPECTIVES}


Que la formation des ingénieurs ne se réduise pas aux sciences et techniques et comprenne aussi une formation en sciences humaines, sociales et économiques (SHSE) se comprend dès lors que l'on prend en considération les activités réelles des ingénieurs et leurs fonctions dans les entreprises. Celles-ci ne se limitent pas au calcul scientifique et la conception et fabrication technique, l'ingénieur est aussi chef de projet, responsable d'équipe, manager, dirigeant d'entreprise... Il peut être confronté à des prises de décision à forts enjeux sociétaux, à d'autres cultures et valeurs que les siennes. Il est pris dans des relations professionnelles où le comportement stratégique des acteurs est souvent central ... Mais la question de cette formation ne va pas sans dire. Par qui est-elle assurée ? Comment est-elle assurée ? Repose-t-elle sur des champs scientifiques? Est-elle adossée à la recherche ? La formation des ingénieurs ouverte sur les SHSE Positionnement de la formation des ingénieurs, par rapport à cette question en France L'intérêt que les Écoles d'ingénieurs en France portent à la formation en sciences humaines, sociales et économiques pour les élèves ingénieurs ne date pas d'aujourd'hui. Ainsi l'INSA de Lyon (Institut National des Sciences Appliquées) avait dès sa création en 1957 ouvert un " centre des humanités », en 1991 le directeur de l'enseignement supérieur des télécommunications crée le groupe "Humanité pour les ingénieurs », en 1992, la journée organisée par la Conférence des Grandes Écoles s'intitule «Sciences humaines et métiers d'ingénieurs, en 1996 avait lieu à Lyon une colloque intitulé "Humanités et Grandes Écoles »... et l’on pourrait continuer à citer des événements et des déclarations marquant l'importance d'une telle formation pour les ingénieurs. Mais son organisation concrète est plus problématique. Si quelques postes statutaires en SHSE existent dans certaines Écoles, le plus souvent les enseignements en SHSE sont confiés à des consultants et intervenants extérieurs qui ne font pas partie du corps des enseignants titulaires des Écoles. N’est-ce pas une façon de dire que malgré les affirmations de bonne intention, on estime que ces enseignements ne font pas partie des mêmes types d'enseignements que les sciences et techniques ? Ces dernières sont aujourd'hui toujours adossées à la recherche qui confère la légitimité scientifique aux enseignements. Qu'en est-il pour les SHSE ? Il serait sans doute « contre-productif » de dire que les ingénieurs ne doivent pas être ouverts aux questions économiques, humaines et sociales, mais comment former à ces questions qui engagent le comportement, la relation à l'éthique, le rapport aux autres ? La façon dont l'enseignement et la formation en SHSE sont assurés dans les Écoles indique sans doute la conception que celles-ci se font de leur légitimité et de leur valeur. La question reste ouverte. Positionnement de la formation des ingénieurs, par rapport à cette question au Québec Au Québec, la profession d'ingénieur est sous l'égide d'Ingénieur Canada, qui est l'organisme centralisateur qui appuie les ordres provinciaux et territoriaux dans la régulation de la profession d'ingénieur. Il représente les ingénieurs canadiens par rapport à de nombreux enjeux touchant notamment le développement durable, la reconnaissance et la mobilité nationale et internationale des ingénieurs. Ingénieur Canada délivre aux ingénieurs leurs permis d’exercice de la profession. À cette fin, Ingénieur Canada a institué en 1965 le Bureau canadien d'agrément des programmes de génie (BCAPG), qui édicte les principes et les normes d'accréditation des programmes universitaires de premier cycle en génie au Canada. Ces normes constituent un cadre général qui assure la qualité de l'enseignement du génie et les compétences des étudiants en regard des enjeux sociaux du moment. La première norme en matière d'études complémentaires, qui remonte à 1977, approuve les études touchant les sciences humaines, sociales et administratives et plus spécifiquement l'économie appliquée à l'ingénierie. Les changements sociaux, technologiques, environnementaux et organisationnels des années 80 ont orienté le BCAPG vers de nouvelles normes touchant plus spécifiquement la question des impacts de la technologie sur l'environnement, les responsabilités morales de l'ingénieur et la maitrise de méthodologies scienti- 
fiques propres aux sciences humaines et sociales. Un mouvement qui, durant les années qui ont suivi, fut appuyé par la communauté des ingénieurs. L’Académie canadienne du génie, la Commission des partenaires du marché du travail et l'Ordre des ingénieurs du Québec se sont de fait positionnés en faveur des cours de sciences sociales et humaines dans les programmes d'ingénierie. À ce jour, toutes les Écoles d'ingénierie québécoises adoptent dans leurs programmes, à différentes intensités, des cours, des modules, voire même des unités institutionnelles d’études complémentaires, dédiés aux sciences humaines et sociales. De plus, l’Ordre des Ingénieurs du Québec publia en 2011 le nouveau référentiel des «qualités» attendues de l’ingénieur promu par Ingénieur Canada. Ainsi, en plus des compétences techniques et scientifiques, toute École d'ingénierie au Canada doit s'assurer que ses étudiants finissants sont des professionnels capables de communiquer et de travailler en équipe. Ils doivent être en mesure d'analyser les impacts du génie sur la société et l'environnement. Ils doivent respecter les principes de l'éthique, de l'économie et des affaires, dans la perspective de l'apprentissage tout au long de la vie. Ces nouvelles normes humaines et sociales de la profession d'ingénieur témoignent du rapprochement qui s'intensifie entre la science, la technologie et la société. On constate de fait que le BCAPG identifie dans son référentiel un nombre de «qualités» humaines et sociales plus important comparativement aux qualités techniques et scientifiques que l'ingénieur doit maitriser dans le cadre de sa profession. Or, peut-on réellement affirmer sur cette base que la question des sciences humaines et sociales est tout autant soutenue au sein de la recherche scientifique dans les institutions d'ingénierie canadiennes? Une question fondamentale, à notre avis, dans la mesure où elle met en cause la qualité de l'enseignement des sciences humaines et sociales appliquées à l'ingénierie... Adossement à la recherche des formations en SHSE dans les Écoles Elle prend une signification particulière lorsqu'on la relie à cette autre question qui nous intéresse ici. Les formations économiques, sociétales et humaines sont-elles soutenues par des activités de recherche en sciences humaines, économiques et sociales dans les Écoles d'ingénieurs? Quelle place les établissements d’enseignement supérieur qui ont en charge la formation des ingénieurs réservent-elles à la recherche en SHSE ? Dans les Écoles les formations scientifiques et technologiques (sciences pour Ingénieurs) sont adossées à la recherche à travers des laboratoires parfois partagés entre établissements. Qu'en est-il pour les SHSE ? Positionnement sur la question au Québec Plusieurs questions restent effectivement ouvertes au Québec quant aux assises scientifiques des cours de sciences sociales et humaines dans les programmes détude universitaire en génie. Dans quelle mesure, par exemple, l'institution supporte-t-elle la recherche scientifique sur la question du rapport entre technologie et société dans la profession de l'ingénieur ? En quoi les cours qui sont offerts dans les programmes universitaires reposent-ils sur des fondements scientifiques à jour ? D'autres questions se posent également par rapport à la qualité de l’enseignement des sciences humaines et sociales. Quelles sont par exemple les qualifications scientifiques, l'expérience et les compétences des enseignants qui dispensent les cours en sciences sociales et humaines ? En quoi l'institution renforce-t-elle l'apport de la recherche scientifique à l'enseignement des sciences sociales et humaines? Position de la CTI en France sur la question En France la Commission des Titres d'Ingénieurs (CTI)[1] qui habilite les Écoles à délivrer le titre d'ingénieur a pris position sur cette question. La CTI fait autorité auprès des Écoles. C’est un organisme indépendant, chargé par la loi française depuis 1935 d'évaluer toutes les formations d'ingénieurs en France et de développer la qualité de ces formations. Elle est composée de 32 membres dont 16 sont issus du monde de l'entreprise et 16 du monde académique. Elle porte aussi un regard critique sur les formations et conseille les établissements. Dans le cahier complémentaire 2010 aux " Références et Orientations " pour l'habilitation des formations d'ingénieur elle note non seulement que "Les pratiques professionnelles des ingénieurs impliquent de façon incontournable la prise en compte des composantes humaines, économiques et sociales de ces métiers dans leur formation ", mais encore que « les formations économiques, sociales et humaines doivent désormais s'appuyer, au même titre que les autres champs de formation, sur des champs scientifiques de référence ». En ce sens, « la qualité des enseignements est soutenue par des activités de recherche en sciences humaines, économique et sociales. » En d'autres termes, la formation aux SHSE, estime la CTI, doit s'appuyer sur une recherche académique reconnue. Elle insiste sur l'intérêt des recherches croisées en sciences pour l'ingénieur et les SHSE qui permettent de mieux explorer les activités de conception des ingénieurs et souligne aussi l'intérêt des recherches sur les formations (didactique, pédagogie...), sur les environnements socioprofessionnels des ingénieurs, les enjeux sociétaux de leurs activités et leur prise de décision. Elle invite chaque Ecole à développer les formations en 
SHSE et la recherche dans ces domaines en les liant à leur projet pédagogique. C'est reconnaitre aussi que l'adossement à la recherche contribuera à légitimer « académiquement » ces formations dont elle souligne à la fois la légitimité et la diversité. Appel à communication C'est précisément l'objectif de ce numéro thématiquede la revue « Phronésis » : faire connaitre la diversité des recherches en SHSE dans les Écoles d'ingénieurs et mettre en évidence lappui que ces recherches fondamentales et finalisées apportent à l'excellence des formations.

\section{Les contributions}

Marianne CHOUTEAU Marie-Pierre ESCUDIE, Joëlle FOREST et Céline NGUYEN proposent une réflexion théorique sur l'artificialisation du notre monde à travers les objets techniques qui représenteraient l'un des piliers de ce qui caractérise notre humanité. Elles font état de la culture technique, en tant que champ interdisciplinaire centré sur l'étude des relations entre technique et société dans la formation des ingénieurs et posent l'hypothèse que cette culture technique devrait en être un élément clé. Relatant une expérience à l'INSA de Lyon les auteures nous invitent à examiner les conditions de réalisation de la culture technique au niveau de l'enseignement, via une recherche interdisciplinaire.

Carole ROBY retrace l'évolution historique de la formation en sciences humaines et sociales (SHS) dans les écoles d'ingénieurs en France ainsi que celle de la mise en place de la recherche en SHS dans ces écoles. Lauteur nous présente le passage progressif des humanités aux SHS en passant par la formation humaine des ingénieurs comme un processus non linéaire. Madame ROBY démontre que les efforts engagés depuis les années 1950 pour développer les formations en SHS, n'ont que très rarement permis de penser la science et la technique en tant que réalités humaines et sociales.

Michel LEJEUNE présente la professionnalisation de l'ingénieur, sous l'angle de la sociologie de la technologie. $S$ 'inscrivant dans la perspective des énoncés de principe et les normes canadiennes d'agrément des programmes universitaires d'ingénierie. La question du développement des technologies (production, diffusion et appropriation) se rattache en tous points aux qualités personnelles et sociales de l'ingénieur auxquelles réfèrent les écoles d'ingénierie et leurs organismes de régulation. La profession d'ingénieur s'enracine d'ailleurs dans des milieux industriels et sociaux de plus en plus sensibles aux dimensions sociales de la technologie, considérant les nouvelles dynamiques qui s’y rattachent en regard des impacts de la technologie sur la société et dans les entreprises.

Jean-Gabriel OFFROY propose une réflexion sur 25 ans de formation aux relations humianes à l'EISTI (Ecole Internationale des Sciences du Traitement de l'Information), à Cergy-Pontoise. Les tensions entre l'informatique et les dimensions humaines du travail d'ingénieur ont graduellement laissé place à une augmentation des heures dédiées la formation aux ressources humaines et une répartition des modules tout au long de la formation.

Christian MICHELOT soutient la nécessité de susciter la créativité dans les écoles d’ingénieurs en présentant deux modèles théoriques qui permettent de mettre en place une activité créatrice. Il démontre que ces processus de créativité sont en fait des processus de déconnexion.

Ce numéro riche et varié propose donc une perspective historique sur la place de la recherche en sciences humaines, économiques et sociales dans les écoles d'ingénieurs, mais aussi un regard sur la formation aux ressources humaines et aux dimensions sociologiques de ces écoles ainsi que des réflexions théoriques sur la culture technique et la créativité. 